\title{
Assessing post-cardiac stress test heart rate recovery and systolic blood pressure recovery in patients suffering from vasovagal syncope
}

\author{
Mehdi Moradi ${ }^{1}$, Maryam Farhadian², Mehdi Kazemi ${ }^{3}$
}

\begin{abstract}
Background and purpose: Syncope is caused by disorder in blood flow to the brain and usually occurs in autonomic system dysfunction. On the other side, heart rate and systolic blood pressure response during cardiac exercise test as well as recovery stage depend upon autonomic nervous system. This research compares heart rate recovery (HRR) and systolic blood pressure recovery (SBPR) following exercise test among patients with vasovagal syncope and normal individuals.

Research methodology: In this cross-sectional descriptive study, 31 patients with vasovagal syncope confirmed by head up tilt test (HUTT) and 32 normal individuals were investigated within 2015- 2017. Systolic and diastolic blood pressures, as well as heart rate were measured for both groups in the first and third minutes post exercise test; and any changes in blood pressure and heart rate were compared.

Findings: $48.39 \%$ and $51.61 \%$ of the patients group were female and male, respectively; and $59.37 \%$ and $40.63 \%$ of control group consisted of female and male. Mean age range in experimental group was 39.31 and 34.45 for control group. Mean HRR and mean SBPR for minute one was higher in patient group than control group $(P$ value $=0.1)$ and $P$ value $=0.12)$ respectively; while, mean HRR and SBPR for minute three showed larger in control group than the patients $(P$ value $=0.31$ and 0.1 ) respectively.

Conclusion: Patients suffering from vasovagal syncope showed different recovery test response than control group due to autonomic system dysfunction.
\end{abstract}

Keywords: syncope, vasovagal, heart rate recovery, blood pressure recovery

\section{INTRODUCTION}

Syncope is a loss of consciousness caused by disorder in blood flow to brain for a short period and spontaneously recovers. It is characterized with fast onset, short duration, and spontaneous recover $(1,2)$. Syncope is a critical clinical issue as it is common, costly, and often disabling, which may even causes loss and hurt. Further, it may be the first warning sign prior sudden cardiac death (2). The annual incidence of syncope is reported 1.3 to 2.7 per 1000 population in year (3). $40 \%$ of adults experience syncope at least for one time (4-6). Several factors cause syncope; however, the most common at any age range is neurally mediated syncope (7). The neutrally mediated syncope (also known as neurocardiogenic, vasodepressor, and vasovagal syncope) has been used to describe a common abnormality in regulation of blood pressure characterized by an abrupt onset of hypotension with or without bradycardia (2).

It is stated that vasovagal syncope caused by a paradoxical reflex begins when ventricular preload is decreased as a result of venous pooling. This reduction leads to decrease cardiac output and blood pressure, which is sensed by atrial baroreceptors. It finally leads to vasodilatation and bradycardia through a set of relevant chain responses by vagus nerve stimulation (2-8).

Patients with vasovagal syncope are usually evaluated by using head up Tilt table test (HUTT).This test is considered abnormal or positive in case patients experience syncope during the test. Positive tilt test includes vasodepressor or

\footnotetext{
MD, Assistant professor of Cardiology, Farshchian Heart Center, Hamadan University of Medical Science, Hamadan, Iran.

2 PhD, Department of Biostatistics, School of Public Health and Research Center for Health Sciences, Hamadan University of Medical Science, Hamadan, Iran

3 MD, Farshchian Heart Center, Hamadan University of Medical Science, Hamadan, Iran.
}

\author{
Correspondence: Mehdi Morda \\ MD, Assistant professor of Cardiology, Farshchian Heart Center, Hamadan University \\ of Medical Science, Hamadan, Iran. \\ E-mail:mmoradi341@yahoo.com
}

Received: 23 Feb 2018, Accepted: 20 April 2018

(C) 2019 by the authors; licensee Modestum Ltd., UK. This article is an open access article distributed under the terms and conditions of the Creative Commons Attribution License (http://creativecommons.org/licenses/by/4.0/). 
cardioinhibitory response and or combination of both. In this test both sympathetic and parasympathethic systems are involved and abnormal response indicates dysfunction of autonomic nervous system.

Sympathetic and parasympathetic system tones also undergo some changes during different stages of cardiac exercise test so as the parasympathetic system is initially inhibited; then, sympathetic system is activated. Once cardiac stress test is stopped, parasympathetic system is dominant at the recovery phase to decrease heart rate gradually.

Since both sympathetic and parasympathetic systems are actively involved in both recovery phase of exercise test and HUTT, assessing patients suffering from vasovagal syncope confirmed by HUTT by exercise test can provide useful and practical information about changes it autonomic nervous system.

OF note, prognostic significance of abnormal HRR is strongly independent from drug usage (even Beta-blocker), maximum level in cardiac stress test, severity of coronary artery involvement in angiogram, treadmill Duck score, ejection fraction, electrocardiographic abnormalities during cardiac stress test, and chest pain (9).

Meanwhile, reduced heart rate at the recovery phase of exercise test is an independent factor to determine prognosis and mortality in these patients (9-11).

Prior studies focused on reduced heart rate in a constant period once cardiac exercise test is completed (12-15). At break time following cardiac stress test, HR must rapidly decreases. Whether the patient is standing or sitting, decreasing of heart rate must be over 12 beats in the first minute and more than 22 beats for the second minute post exercise comparing maximum $H R$ during test.

$\mathrm{HRR}=\mathrm{HRmax}-\mathrm{HR} 1$ or 2 minutes later

Normal blood pressure response to cardiac exercise test is gradual increase in systolic pressure up to $160-200 \mathrm{mmHg}$. However, diastolic blood pressure showed no significant change.

In recovery stage of exercise test, systolic blood pressure gradually decline through activation of parasympathetic and mitigation of sympathetic system. Subsequently, measurement of blood pressure in recovery phase might provide useful clinical findings about function of autonomic systems.

It is noteworthy to emphasize that lack of increase in systolic blood pressure during exercise, decreased in systolic blood pressure or reducing systolic blood pressure to less than break level are regarded abnormal and indicating inadequate increase in cardiac output resulting from left ventricular systolic dysfunction or systemic vascular resistance impairment(9).

The present research is conducted to assess SBP recovery and HR recovery post-cardiac exercise test in patients suffering from vasovagal syncope. The research hypothesized that cardiac exercise test abnormalities, including HR recovery and SBP recovery may be probably associated with vasovagal syncope outbreak. Since vasovagal syncope lacks any definitive treatment, further studies may shed a new light on findings of etiology and treatment.

\section{RESEARCH METHODS}

In this cross-sectional descriptive study, 31 patients suffering from vasovagal syncope confirmed by head up tilt test visited at Farshchian Heart Center, within 2015-2016, and 32 normal individuals (control group) were analyzed and both groups compared in term of blood pressure and heart rate post-cardiac exercise test alterations. Research participants' history was collected; further, all underwent physical examination, ECG, and echocardiography. Research exclusion criteria included patient dissatisfaction to continue the study, taking heart rate or blood pressure effective medications, diagnosed coronary artery disease, prior myocardial infarction history, left ventricular dysfunction, cardiomyopathy, congenital heart disease, left ventricular hypertrophy (LVH), mitral regurgitation, aortic stenosis, artificial cardiac pacemaker, preexcitation syndrome, atrial fibrillation, hypothyroidism and hyperthyroidism, hypertension, chronic obstructive pulmonary disease, malignancy, musculoskeletal or orthopedic disorders, and heart failure history.

\section{Statistical Analysis}

Post-cardiac exercise test HRR and SBPR data for the first and third minutes were collected in patients and normal individuals; then, data were analyzed through SPSS 16. Data normality of systolic blood pressure and heart rate was evaluated by Shapiro-Wilk test. Given data normality, post-cardiac stress test mean HRR for the first and third minute and mean SBPR for the first and third minute in both groups were compared using parametric independent t-test; further, SBPR mean for the first minute was compared using non-parametric Mann-Whitney test. To obtain the proper cut point in order to distinguish patients and normal individuals, Tilt test sensitivity, specificity, positive and negative predictive value, as well as HRR precision for the first minute post-cardiac stress test were measured at several cut points and ROC curve was illustrated. 
Table 1: Post-cardiac stress test HRR and SBPR comparisons at the first and third minutes for patient and control groups

\begin{tabular}{ccc}
\hline & Group & Indicator \\
\hline Control & Patients & HRR $\min 1$ \\
\hline $44.18(2.27)$ & $47.19(2.05)$ & $\mathrm{HRR}_{\min 3}$ \\
\hline $65.75(2.26)$ & $62.74(1.68)$ & $\mathrm{SBPR}_{\min 1}$ \\
\hline $17.59(1.65)$ & $21.87(1.92)$ & $\mathrm{SBPR}_{\min 3}$ \\
\hline $41.34(2.30)$ & $36.74(2.04)$ & \\
\hline
\end{tabular}

Table 2: Comparing HRR $\min 1$ at cut point 47 and Tilt test for syncope patients and normal individuals differentiation

\begin{tabular}{ccccc}
\hline \multirow{2}{*}{ Total } & \multicolumn{3}{c}{ Tilt Test } & Result \\
\cline { 2 - 4 } & Normal & Patient & $47>$ \\
\hline 29 & 13 & 16 & $47 \leq$ \\
\hline 34 & 19 & 15 & Total \\
\hline 63 & 32 & 31 & HRR \\
\hline
\end{tabular}

Table 3: Comparing SBPR $\min 1$ at cut point 18 and Tilt test for syncope patients and normal individuals differentiation

\begin{tabular}{ccccc}
\hline \multirow{2}{*}{ Total } & \multicolumn{2}{c}{ Tilt Test } & \multirow{2}{*}{ Result } \\
\cline { 2 - 4 } & Normal & Patient & $18>$ & $18 \leq$ \\
\hline 29 & 12 & 17 & Total & SBPRmin1 \\
\hline 34 & 20 & 31 & 14 & \\
\hline 63 & 32 & &
\end{tabular}

To attain a proper cut point distinguishing vasovagal patients from normal individuals, many several cut points were created at mean SPBR interval for patient and control groups using one minute post-cardiac stress test SBPR through software. Research significance level is 0.05 .

The present research is conducted with the approval of ethics committee of Hamedan University of Medical Sciences, recommended by medical school under Farshchian Hospital management permission.

\section{RESULTS}

63 individuals (31 patients and 32 as control) participated in this study in which 15 individuals (48.39\%) were male and 16 (51.61\%) were female in experimental patient group; whereas, control group consisted of 19 male (59.37\%) and 13 female (40.63\%) $(P=0.38)$.

Mean age and standard error obtained $39.31 \pm 1.19$ among patient group (minimum 13 years and maximum 73 ). Mean age and standard error of control group measured $34.45 \pm 2.64$, minimum 29 years and maximum 57 years old. Respecting age range, patient and control groups showed no statistically significant difference $(P=0.096)$.

Mean HR recovery and SBP recovery at the first minute after cardiac stress test in patients suffering from vasovagal syncope were larger than control group; however, statistically insignificant ( $P$ value $=0.1$ and 0.12$)$ respectively $($ Table 1 ).

Meanwhile, the third minute post-cardiac stress test HRR and SBPR results revealed less level in vasovagal syncope patients than control group which were statistically insignificant ( $P$ value $=0.31$ and 0.1 ) (Table 1).

To estimate post-cardiac stress test HRR precision distinguishing patients from normal individuals, first minute HRR sensitivity, specificity, positive and negative predictive value were compared with mean and up to three scores larger and three scores lower than the mean; hence, first minute HRR at cut point 47 represented the highest precision (55.56\%) and ROC sub curve level (56\%) (Table 2 and Figure 2).

To attain a proper cut point distinguishing vasovagal patients from normal individuals, many several cut points were created at mean SPBR interval for patient and control groups using one minute post-cardiac stress test SBPR through software. For each cut point, specificity and sensitivity were also obtained in addition to ROC sub curve level; ultimately, systolic blood pressure recovery showed the largest ROC sub-curve area (0.587) and diagnosis precision (53.97\%) at cut point $18 \mathrm{mmHg}$ (Table 3, and Figure 2). 


\section{ROC Curve}

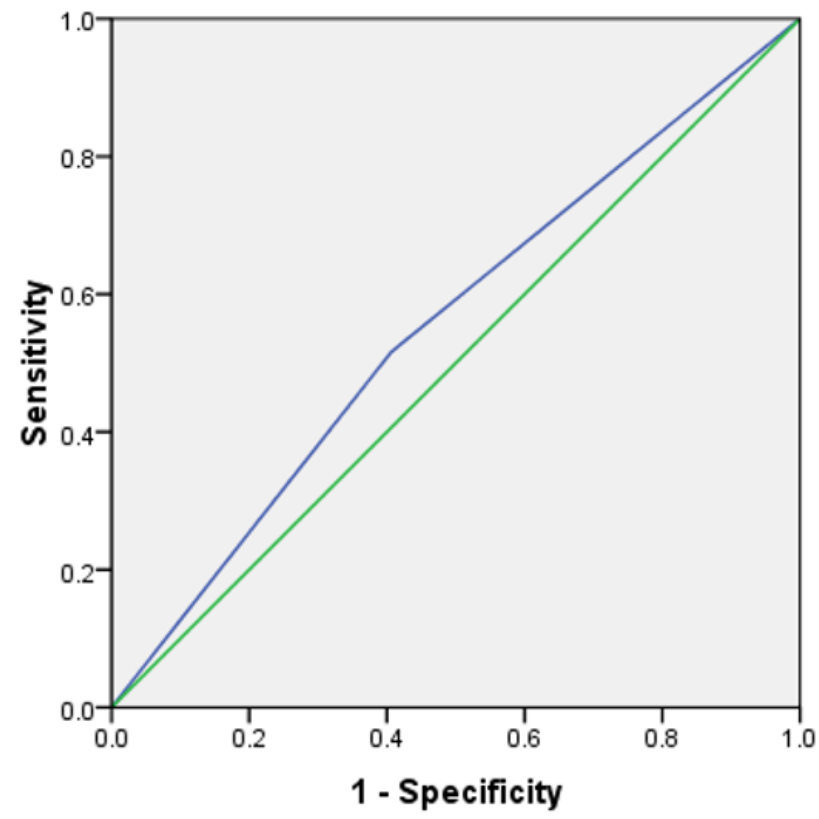

Diagonal segments are produced by ties.

Figure 1 Post-cardiac stress test HRR ROC curve one minute later at cut point 47

\section{ROC Curve}

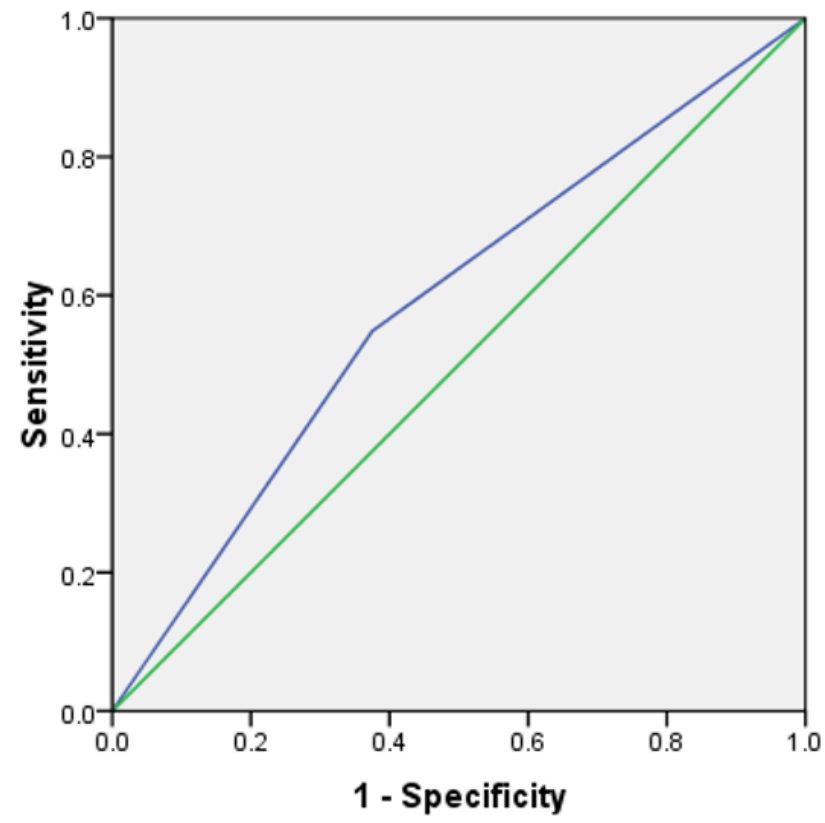

Diagonal segments are produced by ties.

Figure 2: Post-cardiac stress test SBPR ROC curve one minute later at cut point 18

\section{DISCUSSION}

Syncope is a transient loss of consciousness due to transient global cerebral hypoperfusion characterized by rapid onset, short duration, and spontaneous complete recovery (18).

The most common causes of syncope in all age groups are neurally mediated disorders $(1,7)$. These are benign conditions with no associated increase in mortality that are characterized by vasodilatation or vagally driven bradycardia 
or asystole, which cause profound systemic hypotension and consequent dizziness, presyncope, and syncope (5). It is stated that withdrawal of peripheral sympathetic tone and an increase in vagal tone can result in vasovagal syncope (2).

This syncope often has benign course, however, due to recurrence and lack of specific treatment, it can be so debilitating for patients.

Patients with vasovagal syncope are usually evaluated by using head up Tilt table test (HUTT) and according to results of abnormal test, the patients are classified to three groups, vasodepressor, cardioinhibitory and mixed type.

The heart rate and systolic blood pressure increase during exercise test because of an increase in sympathetic tone and a decrease in parasympathetic tone. At the cessation of exercise, under normal circumstances, the reverse process occurs. That is, first parasympathetic tone increases and then sympathetic tone decreases.

Since changes in the heart rate and blood pressure during recovery phase of exercise test and abnormal response during HUTT depend largely on tone of sympathetic and parasympathetic system, evaluation of patients suffering from vasogal syncope with exercise test may provide some valuable information.

To the best of our knowledge there is a very few if any study about abnormalities of exercise test in patients with vasovagal syncope.

HRR as a parasympathetic tone index was only evaluated in Kocabas et al (17) to diagnose neurocardiogenic syncope. In this study, heart rate recovery was significantly higher in patients suffering from vasovagal syncope than control group for the first min. The authors deduced that first minute recovery may contribute in neurocardiogenic syncope diagnosis (17). Accordingly, the present research results also implied higher HRR in vasovagal patients than control individuals at the first minute recovery phase consistent with Kocabas finding.

According to the research results, post-cardiac stress test mean SBPR and mean HRR at the first minute were higher in patient group than control group (regarding parasympathetic system contribution) indicating high parasympathetic tone in individuals suffering from vasovagal syncope. It means that blood pressure and heart rate rapidly decline in patients suffering from vasovagal syncope than normal individuals.

On the other hand, higher HRR and SBPR at the third minute for control group comparing patients group indicates that in patients group tone of sympathetic system decrease with greater extent than control group after three minute of exercise cessation.

Post-cardiac stress test SBPR, in the present research, was larger in patients than normal ones after one minute. SBPR at cut point $=18 \mathrm{mmHg}$ showed the widest ROC sub-curve area (0.587) and diagnosis precision (53.97\%). SBPR diagnosis precision at the first minute was even larger than HRR in differentiating vasovagal syncope and normal individuals.

In short, this study in principle confirms that autonomic nervous system plays an important role not only in pathogenesis of vasovagal syncope but also different response of these patients to recovery stage of exercise test.

\section{CONCLUSION}

This study shows physiologic changes in sympathetic and parasympathetic tone immediately after cessation of exercise test (recovery phase) is noticeably different in patients suffering from vasovagal syncope compare to normal individual. Moreover, HRR and SBPR impairments, especially at the first minute recovery phase confirm autonomic system dysfunction in patients enduring vasovagal syncope.

\section{Research Limitations and Recommendations}

Mean age range was higher in control group than patient group. Although, they showed no statistically significant difference; equal mean age range may improve study strength. Patient group, in this study, consisted of the individuals previously experience vasovagal syncope during head up Tilt test. But control group lacked Tilt test assessment before study.

False positive result of head up Tilt test is another limitation should be taken into account.

\section{ACKNOWLEDGEMENTS}

The authors would like to express gratitude to the deputy of research and technology of Hamadan University of Medical Sciences, clinical research department of Farshchian medical education heart center for their cooperation and advices. It is also necessary to thank to non-invasive testing unit staff. The paper is extracted from assistant dissertation of Hamedan University of Medical Sciences in the field of cardiology. 


\section{REFERENCES}

1. Kasper D, Fauci A, Hauser S, Longo D, Jameson JL, Loscalzo J. Harrison`s principle of Internal Medicine.19th Edition, Chapter 27; Syncope. 2015.

2. Mann D, Zipes D, Libby P, Bonow R. Braunwald's Heart Disease. 10th Edition, Chapter 40; Hypotension \& Syncope. 2015.

3. Chen L, Chen MH, Larson MG, Evans J, Benjamin EJ, Levy D. Risk factors for syncope in a community-based sample (the Framingham Heart Study). Am J Cardiol. 2002;85(10):1189-93. https://doi.org/10.1016/S00029149(00)00726-8

4. Alboni $P$, Brignole M, Menozzi C, Raviele A, Del Rosso A, Dinelli M et al. Diagnostic value of history in patients with syncope with or without heart disease. J Am College Cardiol. 2001;37(7):1921-8. https://doi.org/10.1016/S0735-1097(01)01241-4

5. Parry SW, Tan MP. An approach to the evaluation and management of syncope in adults. BMJ. 2010;340:c880. https://doi.org/10.1136/bmj.c880

6. Olde Nordkamp LR, van Dijk N, Ganzeboom KS, Reitsma JB, Luitse JS, Dekker LR, et al. Syncope prevalence in the ED compared to general practice and population: a strong selection process. Am J Emerg Med. 2009;27(3):2719. https://doi.org/10.1016/j.ajem.2008.02.022

7. Saedi S, Oraii S, Hajsheikholeslami F. A Cross Sectional Study on Prevalence and Etiology of Syncope in Tehran Acta Medica Iranica. 2013;51(10):715-9.

8. Fuca $G$, Dinelli $M$, Suzzani $P$, Scarfo $S$, Tassinari $F$, Alboni P.The venous system is the main determinant of hypotension in patients with vasovagal syncope. Europace, 2006;8:839-45. https://doi.org/10.1093/europace/eul095

9. Mann D, Zipes D, Libby P, Bonow R. Braunwald`s Heart Disease. 10th Edition, Chapter 13; Exercise Stress Testing. 2015.

10. Yawn BP, Ammar KA, Thomas R, Wollan PC. Test-Retest Reproducibility of Heart Rate Recovery After Treadmill Exercise. Ann Fam Med 2003;1:236-241. https://doi.org/10.1370/afm.37

11. Watanabe J, Thamilarasan M, Blackstone E, Thomas J and Lauer M. Heart Rate Recovery Immediately After Treadmill Exercise and Left Ventricular Systolic Dysfunction as Predictors of Mortality. Circulation. 2001;104:19111916.

12. Desai MY, De la Pena-Almaguer E, Mannting F. Abnormal heart rate recovery after exercise as a reflection of an abnormal chronotropic response. Am J Cardiol. 2001;87:1164-9. https://doi.org/10.1016/S0002-9149(01)014874

13. Dresing TJ, Blackstone EH, Pashkow FJ, Snader CE, Marwick TH, Lauer MS. Usefulness of impaired chronotropic response to exercise as a predictor of mortality, independent of the severity of coronary artery disease. Am J Cardiol. 2000;86:602-9. https://doi.org/10.1016/S0002-9149(00)01036-5

14. Cole CR, Foody JM, Blackstone EH, Lauer MS. Heart rate recovery after submaximal exercise testing as a predictor of mortality in a cardiovascularly healthy cohort. Ann Intern Med. 2000;132:552-555. https://doi.org/10.7326/0003-4819-132-7-200004040-00041

15. Shetler K, Marcus R, Froelicher VF, et al. Heart rate recovery: validation and methodologic issues. J Am CollCardiol. 2001;38:1980-7. https://doi.org/10.1016/S0735-1097(01)01652-7

16. Cole C, Blackstone E, Pashikow F, Snader C and Lauer M. Heart-Rate Recovery Immediately After Exercise As A Predictor Of Mortality. N Engl J Med 1999;341:1351-7. https://doi.org/10.1056/NEJM199910283411804

17. Kocabas $U$, Kaya EB, Aytemir $K$, Yorgun $H$, Kepez A, Aksoy $H$, et al. A novel method for the diagnosis of neurocardiogenic syncope: heart rate recovery index. Cardiology. 2009;114(1):50-5. https://doi.org/10.1159/000212079

18. Moya A, Sutton R, Ammirati F, et al. Guideline for the diagnosis and management of syncope 2009.Eur Heart J 30:2631, 2009.

$\diamond \diamond \diamond \diamond \diamond \diamond \diamond$

http://www.ejgm.co.uk 